\title{
Comparison of Two Methods for Estimating the Tip Position of a Nasogastric Feeding Tube: A Randomized Controlled Trial
}

\author{
Tim Torsy, RN, MSc ${ }^{1}$; Renée Saman, RN, CNS, MSc ${ }^{1,2}$; Kurt Boeykens, RN, CNS, \\ $\mathrm{MSc}^{2}$; Ivo Duysburgh, $\mathrm{MD}^{2}$; Nele Van Damme, RN, $\mathrm{MSc}^{3}$; \\ and Dimitri Beeckman, RN, MSc, PhD $^{3}$
}

\begin{abstract}
Background: Several studies have shown that the nose-earlobe-xiphoid distance (NEX) is inadequate to estimate the insertion length of nasogastric tubes. An alternative approach tested in these studies, which leads to $>90 \%$ well-placed nasogastric tubes, used a corrected calculation of the NEX: $($ NEX $\times 0.38696)+30.37$. The primary aim of this study was to determine whether using the corrected NEX was more successful than the NEX in determining the insertion length. The secondary aim was to investigate the likelihood to successfully obtain gastric aspirate. Methods: Adult patients in a general hospital $(\mathrm{N}=215)$ needing a nasogastric tube were randomized to the control (NEX) or intervention group (corrected NEX). Tip positioning was verified using X-ray. Correct tip positioning was defined as between 3 and $10 \mathrm{~cm}$ under the lower esophageal sphincter (LES). Results: In $>20 \%$ of all patients, both methods underestimated the required tube length for correct positioning. The NEX showed an overestimation (17.2\%) of the insertion length ( $>10 \mathrm{~cm}$ under the LES) compared with the corrected NEX $(4.8 \%)$. There was no difference $(P=0.938)$ between the 2 groups in obtaining gastric aspirate $(55.6 \%$ vs $56 \%$ ). However, correct tip positioning resulted in a fourfold increase of obtaining gastric aspirate. Conclusions: Both methods resulted in a high risk of incorrectly placed tubes due to malposition of the tip near the LES or distal esophagus. This may increase the risk of reflux and pulmonary aspiration. Based on these results, the development of more reliable methods requires consideration. (Nutr Clin Pract. 2018;00:1-8)
\end{abstract}

\section{Keywords}

enteral nutrition; gastrointestinal intubation; nasogastric tube; nutritional support; patient safety; X-rays

\section{Introduction}

Enteral feeding via nasogastric tube is a frequently used short-term method for patients who cannot eat or whose oral nutrition intake is insufficient. In adults, the stomach is a $J$-shaped organ with a greater curvature of $34 \mathrm{~cm}$, a maximum transverse diameter of $10 \mathrm{~cm}$, a pyloric sphincter diameter of $1.2 \mathrm{~cm}$, and a capacity of 0.9 litres. ${ }^{1}$ A correct position of the tip of the nasogastric tube inside the stomach is required to ensure the safe administration of nutrients. ${ }^{2,3}$ Correct positioning will also prevent reflux and aspiration of nutrients into the lungs. An incorrect gastric tip position due to underestimation of the tube may lead to feeding remaining in the esophagus because most tubes have several lateral openings proximal of the distal tip opening. Conversely, overestimation (leading to a tube segment located too deep into the stomach) can cause curling of the tube inside the stomach or even migrating the tube back into the esophagus or beyond the pylorus into the duodenum. Incorrect positioning may also prevent obtaining an aspirate for $\mathrm{pH}$ measurement or gastric residual volume. ${ }^{4,5}$

Although pulmonary aspiration is 1 of the most common complications in enterally fed patients, reliable prevalence data about aspiration are difficult to determine due to vague definitions, poor assessment methods, and varying levels of clinical recognition. Pulmonary aspiration of feeding can be caused by either pulmonary misplacement of the tube or incorrect positioning of the tube in the gastrointestinal tract. In 2005, incorrectly placed nasogastric tubes in general were first recognized in England by the National Patient Safety Agency (NPSA) as a patient safety issue. Between September 2005 and March 2010, there were 21 deaths and 79 reported cases in England of harm related

From the ${ }^{1}$ Department of Nursing, Odisee University College, Brussels, Belgium; ${ }^{2}$ AZ Nikolaas General Hospital, Sint-Niklaas, Belgium; and ${ }^{3}$ University Centre for Nursing and Midwifery, Ghent University, Ghent, Belgium.

Financial disclosure: None declared.

Conflicts of interest: None declared.

This article originally appeared online on $\operatorname{xxxx} 0,0000$.

Corresponding Author:

Torsy Tim, RN, MSc, Odisee University College, Department of Health (Nursing and Midwifery), Hospitaalstraat 23, 9100

Sint-Niklaas, Belgium.

Email: tim.torsy@odisee.be 
to feeding through misplaced nasogastric tubes. In 2009, inadequate feeding related to a misplaced nasogastric tube became a never event, which is defined as a serious, largely preventable patient safety incident that should not occur if the available preventative measures have been implemented by healthcare providers. Despite all guidance, during 20092010 there were 41 never events reported to the NPSA in which a misplaced nasogastric tube was not detected prior to use. Even at this time, misplaced nasogastric tubes are never events: Between September 2011 and March 2016, a total of 95 incidents were reported. These incidents show that, despite the sensitization of all stakeholders, risks to patient safety persist. ${ }^{6}$

Most often, the nose-earlobe-xiphoid (NEX) method is used to determine the insertion length of a nasogastric tube. This measures the length from the tip of the nose to the earlobe to the xiphoid. ${ }^{7,8}$ This simple and popular method was introduced by Royce, Tepper, Watson, and Day in 1951. They described a 6-month experience of estimating the appropriate tube length using external anatomical reference points in neonates. Subsequently, the NEX method was recommended as a gold standard for neonates, children, and adults in nursing textbooks without further research or reflection. The reliability of the NEX method in both adults and pediatric populations, however, has been questioned several times. ${ }^{8}-10$ Using the NEX, Ziemer and Carroll noticed that a deeper insertion was often needed to obtain a gastric aspirate. ${ }^{7}$

After a study on healthy volunteers and a postmortem study including 99 cases, Hanson described alternative formulas to calculate the nasogastric tube length in a more reliable way. ${ }^{2}$ Two formulas were proposed as a correction of the NEX (method 1 and 2), and 1 formula was based on body length (method 3):

$$
\begin{aligned}
& \text { Method 1: }([\mathrm{NEX}-50 \mathrm{~cm}] / 2)+50 \mathrm{~cm} \\
& \text { Method 2: }(\mathrm{NEX} \times 0.38696)+30.37 \\
& \text { Method 3: }(\text { body length } \times 0.20239)+17.07
\end{aligned}
$$

Method 2 resulted in more nasogastric tubes being placed correctly inside the stomach $(92.3 \%$ vs $91.35 \%)$, with a tip position ranging from 1 to $10 \mathrm{~cm}$ under the lower esophageal sphincter (LES) being optimal. ${ }^{2}$ For that reason, method 2 was selected as the experimental intervention in this current randomized controlled trial. Ellett et al already had reported that the minimum insertion length of the nasogastric tube corresponds to the distance from the tip of the nose to the gastroesophageal junction, thereby adding the distance from the tip of the tube to the most proximal lateral opening on the tube to ensure that each lateral opening was located inside the stomach. ${ }^{3}$ The actual required length of the nasogastric tube according to either control or intervention group was determined just before the placement procedure starting from the distal tip opening of the tube. To consider a tube as correctly placed inside the stomach, the presence of the lateral openings was taken into account (as described in the interventions and outcomes section of this article). The target population of this study were adults because a method for newborns and children (using the distance between the tip of the nose to the earlobe to the mid-umbilicus) already exists. ${ }^{9,10}$

The primary aim of this first prospective randomized trial was to determine whether the use of the Hanson formula $($ NEX $\times 0.38696)+30.37$ was more successful than using the NEX method. The secondary aim was to investigate the likelihood to obtain a gastric aspirate to perform $\mathrm{pH}$ measurement.

\section{Methods}

\section{Study Design}

This study was designed as a blinded prospective randomized trial. The data were collected between December 2015 and September 2016 in a Belgian general hospital on both intensive care units (ICU) and non ICUs.

\section{Participants}

Patients were eligible to participate if they met the following criteria: adult (aged $\geq 18$ years) and a medical indication for nasogastric tube feeding. Patients were excluded when the xiphoid was not palpable or if they had documented surgical or anatomical abnormalities of the esophagus or stomach (eg, hiatus hernia). Written informed consent was obtained from all patients or their legal representatives in case of diminished awareness or coma.

Participants were randomized to either the control or the intervention group using block randomization. ${ }^{11}$ Randomization was performed by an investigator with no clinical involvement in the trial. A randomization list with 2 groups (control and intervention group), block sizes of 6 patients, and an actual list length of 240 participants (control group and intervention group) was created.

After assignment to 1 of the study groups, blinding was performed at the following levels:

Included patients were kept blinded to their allocation.

Data collectors (clinical nutrition nurse specialists) could not be blinded. They were aware of the allocated arm to perform the appropriate intervention.

Outcome adjudicators (radiologists) were also kept blinded to minimize bias and maximize the validity of the results.

\section{Interventions and Outcomes}

Two experienced clinical nutrition nurse specialists independently measured the NEX distance (Figure 1) in all patients. 


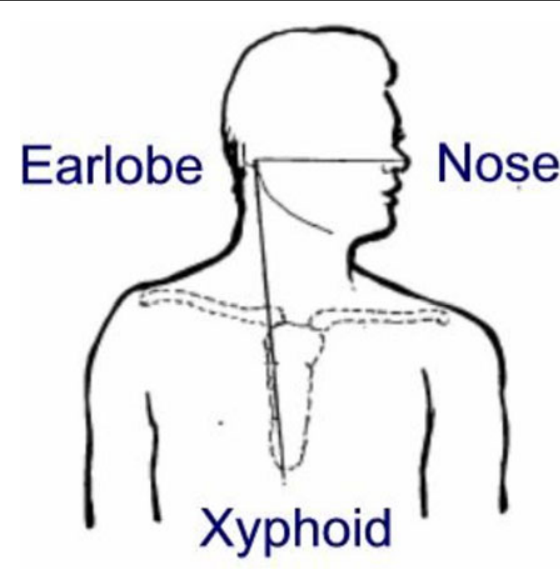

Figure 1. NEX distance.

NEX, nose-earlobe-xiphoid distance.

The 2 measurements were taken within a short space of time according to the following procedure: the patient was in supine position with the chest at an angle of $30^{\circ}$ and facing forward. The chest was uncovered, and the xiphoid was marked. A measuring tape with an accuracy up to half a centimeter was directed in a straight line from the tip of the nose to the earlobe and then to the marked xiphoid (Figure 1). A nasogastric tube was subsequently inserted.

The nasogastric tube used in the study was a polyurethane radiopaque feeding tube (Nutricia Flocare ENFit; CH10 or CH14, total length $110 \mathrm{~cm}$ ), manufactured by Danone Trading B.V., Schiphol, The Netherlands and consisting following characterisitcs: An internal guidewire, a distal opening in the tip, and lateral openings up to $2 \mathrm{~cm}$ from the distal tip. The guidewire ended $5 \mathrm{~cm}$ before the distal tip opening. Newly placed tubes were inserted by the 2 clinical nutrition nurse specialists according to the randomization criteria. If the tubes were already in situ but not yet used for tube feeding, they were only repositioned but not replaced, again according to the randomization criteria. A safety margin of $1 \mathrm{~cm}$ was added so a correct tip position of the nasogastric tube inside the stomach was defined as somewhere between 3 and $10 \mathrm{~cm}$ under the LES. After insertion or repositioning, the tube was fixed to the nose with a fixation tape for nasal tubes manufactured by ConvaTec, Deeside, UK (ConvaTec Naso-Fix).

A gastric aspirate was obtained immediately after insertion or repositioning of the nasogastric tube because $\mathrm{pH}$ measurement is considered an alternative bedside method to X-ray to check the position of a nasogastric tube.11 A large syringe $(60 \mathrm{~mL})$ with an ENFit (Danone Trading B.V., Schiphol, The Netherlands) connection was used for aspiration. An initial attempt of obtaining gastric aspirate occurred before the removal of the guidewire. If no aspirate could be obtained, an extra attempt was made 1 hour after

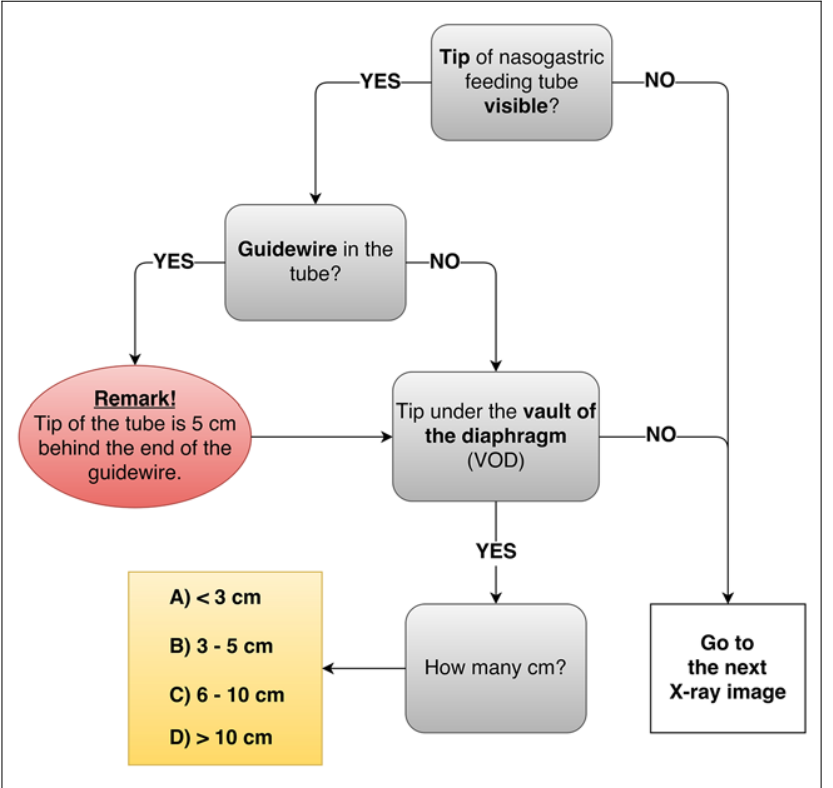

Figure 2. Flowchart for evaluating the X-ray images. $\mathrm{Cm}$, centimeter; VOD, vault of the diaphragm.

removal of the guidewire. Gastric aspirate was tested for $\mathrm{pH}$ using $\mathrm{pH}$ color-indicator strips ( $\mathrm{pH} 2.0-9.0)$ with a $0.5 \mathrm{pH}$ interval scale (Merck KGaA, Darmstadt, Germany). If an aspirate could be obtained, a $\mathrm{pH}$ value $\leq 5.5$ indicated that it was indeed gastric aspirate. ${ }^{12}$

A chest X-ray of the patient, positioned in supine position as described above, was taken in situ to determine the tip position of the tube. Preferably, the guidewire was left inside or put back inside the tube to increase the visibility of the nasogastric tube on X-ray. The chest X-ray images covered the area from the apex to the base of the lungs, including the diaphragm and the stomach below. All of the images were retrieved from the hospital picture archiving and communication system, processed and anonymized using DicomCleaner (PixelMed Publishing, Bangor, PA), and then evaluated on medical imaging monitors.

\section{Instruments}

The chest X-ray images were independently evaluated by 3 different radiologists using a predefined evaluation flowchart (Figure 2). The authors and the 3 participating radiologists used the vault of the diaphragm as the reference point for the location of the LES in the assessment of the images because the LES itself is not very clear on an X-ray.

"Obtainment of gastric aspirate" was considered as negative after 2 attempts (as described above) without retrieving gastric content within 1 hour after insertion. A few drops of the aspirate were placed on a $\mathrm{pH}$ indicator strip. 


\section{Statistical Methods}

Statistical analyses were conducted using SPSS 24.0 (IBM Corporation, Armonk, NY). Categorical variables were presented as frequencies (percentages); normally distributed continuous variables were described using means and standard deviations (SD); and between-group comparisons were performed using independent samples $t$ test. Comparison of categorical data between groups was performed using $\chi^{2}$ test.

The interobserver agreement between the 3 independent radiologists was assessed using $\kappa$ statistics. Observer agreement was defined as 2 or more concurring interpretations of the X-ray being tip $<3 \mathrm{~cm}$ under the LES, between 3 and $10 \mathrm{~cm}$ under the LES, $>10 \mathrm{~cm}$ under the LES, or tip not visible on X-ray. To obtain an overall $\kappa$ value of the assessments, Fleiss $\kappa$ was calculated. The classification system used by Coblentz et al was used to define the strength of agreement for the $\kappa$ coefficient. ${ }^{13}$

The association between the study group and the outcome was controlled for age, gender, body length, and cognitive awareness using a multivariate logistic binary regression analysis. The variable "gastric aspirate obtained" was added to the multivariate model to analyze how this variable was related to the correct positioning of the nasogastric tube. Hosmer-Lemeshow statistic was calculated as a measure of model fit. A significance level of $P<0.05$ was applied.

\section{Ethical Statement and Trial Registering}

All patients (or their legal representative in case of diminished awareness or coma) gave informed consent. Collecting the informed consents was done by the clinical nutrition nurse specialists. Patients or their representatives who were unable or unwilling to sign the written informed consent were excluded from the study.

The study protocol was approved by the Review Committee for Medical Ethics from AZ Nikolaas (East Flanders, Belgium) (EC15053). The study was registered at ISRCTN.com (ISRCTN18322407).

\section{Results}

\section{Patient Characteristics}

A total of 215 patients participated in the study. The quality of the X-ray images of 32 patients was insufficient to allow conclusive determination of the position of the tip of the nasogastric tube. Although the nasogastric tubes were visible on all X-ray images, the images had poor margin settings and thus the tip was not visible on the image and could not be evaluated. Therefore, after the procedure of taking X-ray images, data of only 183 persons were used for further analysis: 99 patients were included in the control group and 84 patients in the intervention group.
Characteristics of the control and intervention group were comparable $(P>0.05)$, as shown in Table 1 .

\section{Tip Position and Gastric Aspirate}

An overall significant difference $(P=0.032)$ was demonstrated between the 2 groups regarding the positioning of the tip. The tip of the nasogastric tube was situated $<3 \mathrm{~cm}$ under the LES in $20.2 \%$ of the tubes in the control group and in $22.6 \%$ in the intervention group $(P=0.691)$. A significant difference $(P=0.009)$ concerning overestimation of the tip position was found between the control group $(17.2 \%)$ and the intervention group (4.8\%). There was no statistically significant difference $(P>0.05)$ between both methods for external length determination and the chance of successfully obtaining gastric aspirate through the nasogastric tube. Absolute values, percentages, and $P$ values are shown in Table 2. The interobserver reliability of the X-ray evaluation between the 3 radiologists indicated acceptable agreement $(\kappa=0.44,95 \%$ confidence interval [CI], 0.39-0.49).

Figure 3 shows the distribution of the nasogastric tube insertion lengths using either the NEX method or Hanson's formula-corrected NEX distance. Using the NEX method, the mean insertion length was $51.62 \mathrm{~cm}$ (SD 3.79) and the interquartile range (IQR) was 4 with a minimum insertion length of $40.5 \mathrm{~cm}$ and a maximum insertion length of $59.5 \mathrm{~cm}$. Using $(\mathrm{NEX} \times 0.38696)+30.37$, the mean insertion length was $50.50 \mathrm{~cm}$ (SD 1.62) and an IQR of 1.95 with a minimum insertion length of $43.5 \mathrm{~cm}$ and a maximum insertion length of $53.0 \mathrm{~cm}$. The distribution of the insertion lengths was significantly lower $(P=0.009)$ using Hanson's formula-corrected NEX distance.

\section{Influencing Factors}

Five potential influencing factors in aiming for correct gastric tip positioning were analyzed in a multivariate binary logistic regression analysis: age, gender (male/female), body length, method of length determination ("NEX"/"(NEX $\times 0.38696)+30.37$ ") and cognitive awareness level (aware/diminished awareness/comatose) (Table 3). The binary outcome variable was "tip position of the nasogastric tube inside the stomach" $(<3 \mathrm{~cm}$ under LES $=$ incorrect position; $\geq 3 \mathrm{~cm}$ under LES = correct position). The variables "weight" and "body mass index (BMI)" were not included in the model due to the large number of missing values ( 81 of $183,44.3 \%)$ and their mutual multicollinearity. The variable "gastric aspirate after placement (yes/no)" was also included in the model to explore the differences between the 2 groups concerning this variable. The model resulted in a significant association $(P=0.001)$ between the outcome variable "tip position" and the obtainment of gastric aspirate (odds ratio 4.109, 95\% CI 1.785-9.458). There is no discrepancy between the model predicted and 
Table 1. Characteristics of Participants $(\mathrm{N}=183){ }^{\mathrm{a}}$

\begin{tabular}{|c|c|c|c|c|}
\hline Characteristics & $\begin{array}{l}\text { Control Group } \\
(\mathrm{n}=99)\end{array}$ & $\begin{array}{l}\text { Intervention Group } \\
\qquad(\mathrm{n}=84)\end{array}$ & $P$ Value & $\begin{array}{c}\text { No Data Available } \\
\text { no. }(\%)\end{array}$ \\
\hline \multicolumn{5}{|l|}{ Demographic variables } \\
\hline Age, mean $\pm S D$, years & $72.39 \pm 12.33$ & $70,62 \pm 12.65$ & 0.339 & \\
\hline \multicolumn{5}{|l|}{ Gender } \\
\hline Male, no. $(\%)^{\mathrm{a}}$ & $52(52.5)$ & $48(57.1)$ & 0.532 & \\
\hline Female, no. $(\%)^{\mathrm{a}}$ & $47(47.5)$ & $36(42.9)$ & 0.532 & \\
\hline \multicolumn{5}{|l|}{ Clinical variables } \\
\hline Body length, mean $\pm \mathrm{SD}$, cm (no.) & $165.37 \pm 9.09(97)$ & $164.83 \pm 8.22(84)$ & 0.675 & $2(1.1)$ \\
\hline Weight, mean $\pm \mathrm{SD}, \mathrm{kg}$ (no.) & $70.59 \pm 17.99(61)$ & $66.36 \pm 14.52(41)$ & 0.193 & $81(44.2)$ \\
\hline $\mathrm{BMI}$, mean $\pm \mathrm{SD}, \mathrm{kg} / \mathrm{m}^{2}$ (no.) & $25.63 \pm 5.74(61)$ & $24.35 \pm 5.01(41)$ & 0.249 & $81(44.2)$ \\
\hline Use of antacids, no. $(\%)^{\mathrm{b}}$ & $78(78.8)$ & $61(72.6)$ & 0.407 & $3(1.6)$ \\
\hline \multicolumn{5}{|l|}{ Unit } \\
\hline ICU, no. $(\%)^{\mathrm{b}}$ & $50(50.5)$ & $44(52.4)$ & 0.800 & \\
\hline Non-ICU, no. $(\%)^{\mathrm{b}}$ & $49(49.5)$ & $40(47.6)$ & 0.800 & \\
\hline \multicolumn{5}{|l|}{ Cognitive awareness level } \\
\hline Aware, no. $(\%)^{\mathrm{b}}$ & $43(43.4)$ & $29(34.5)$ & 0.219 & \\
\hline Diminished awareness, no. $(\%)^{\mathrm{b}}$ & $8(8.1)$ & $14(16.7)$ & 0.075 & \\
\hline Comatose, no. $(\%)^{\mathrm{b}}$ & $48(48.5)$ & $41(48.8)$ & 0.965 & \\
\hline
\end{tabular}

BMI, body mass index; ICU, intensive care unit; no., number; SD, standard deviation.

${ }^{a}$ Data collected by clinical nutrition nurse specialists

${ }^{b}$ Percentages within group

Table 2. Localization of Nasogastric Tubes in Both Groups and Obtainment of Gastric Aspirate $(\mathrm{N}=183)$.

\begin{tabular}{|c|c|c|c|}
\hline & Control Group $(\mathrm{n}=99)$ & Intervention Group $(n=84)$ & $P$ Value \\
\hline \multicolumn{4}{|l|}{ Tip positioning } \\
\hline$<3 \mathrm{~cm}$ under LES, no. $(\%)$ & $20(20.2)$ & $19(22.6)$ & 0.691 \\
\hline 3-10 cm under LES, no. (\%) & $62(62.6)$ & $61(72.6)$ & 0.151 \\
\hline$>10 \mathrm{~cm}$ under LES, no. $(\%)$ & $17(17.2)$ & $4(4.8)$ & 0.009 \\
\hline Obtaining gastric aspirate, ${ }^{\mathrm{a}}$ no. $(\%)$ & $55(55.6)$ & $47(56.0)$ & 0.938 \\
\hline
\end{tabular}

LES, lower esophageal sphincter; no., number.

${ }^{a}$ After placement of the tube.

the effectively observed probabilities (Hosmer-Lemeshow 2.228, degrees of freedom $8, P=0.973$ ).

\section{Discussion}

This study is the first prospective randomized trial in which the reliability of the NEX method and Hanson's formula has been tested. Both methods are inaccurate when aiming for a correct tip position of 3 or more $\mathrm{cm}$ under the LES. When using the NEX, the range of tip positions is larger compared with Hanson's formula (Figure 3). The chance of obtaining an aspirate was low (around 56\%) in both groups (Table 2).

One of the key findings from this study was that in both trial groups in approximately 1 of 5 patients $(20.2 \%$ in the control and $22.6 \%$ in the intervention group), the tip of the tube was located $<3 \mathrm{~cm}$ under the LES. This means that at least 1 lateral opening of the nasogastric tube was located in the esophagus or at the same height as the LES. This can

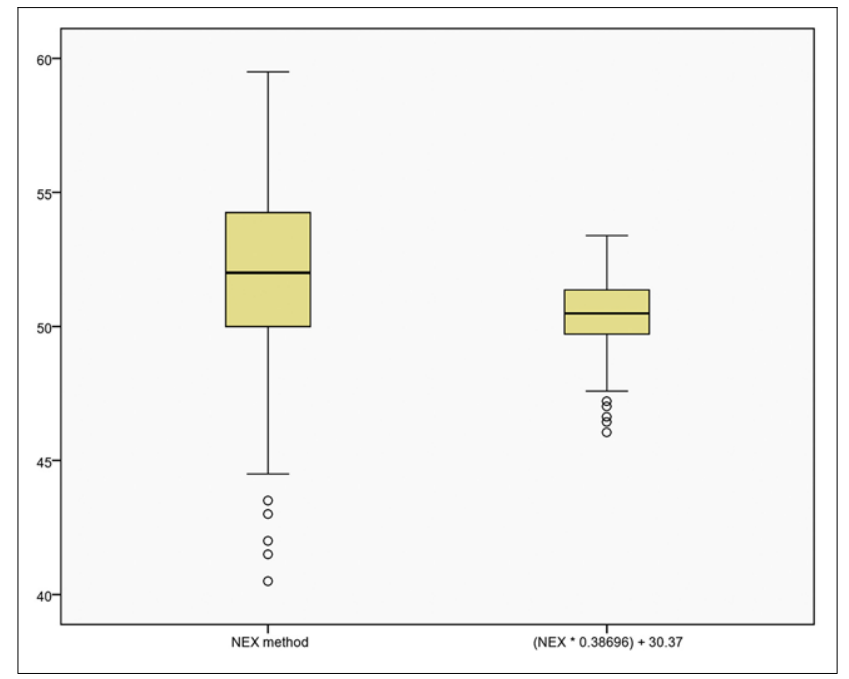

Figure 3. Boxplot describing the distribution of insertion lengths (in centimeters).

NEX, nose-earlobe-xiphoid distance. 
Table 3. Multivariate Binary Logistic Regression Between Possible Influencing Factors in Aiming for a Correct Tip Position.

\begin{tabular}{|c|c|c|c|c|c|c|}
\hline & $\beta$ Coefficient & Standard Error & Wald Statistic & $d f$ & OR $(95 \% \mathrm{CI})$ & $P$ Value \\
\hline Age & -0.007 & 0.020 & 0.135 & 1 & $0.993(0.956-1.032)$ & 0.714 \\
\hline Gender & 0.614 & 0.555 & 1.222 & 1 & $1.847(0.622-5.481)$ & 0.269 \\
\hline \multicolumn{7}{|l|}{ Male $^{a}$} \\
\hline \multicolumn{7}{|l|}{ Female } \\
\hline Body length & 0.031 & 0.035 & 0.779 & 1 & $1.031(0.963-1.103)$ & 0.377 \\
\hline Gastric aspirate & 1.413 & 0.425 & 11.037 & 1 & $4.109(1.785-9.458)$ & 0.001 \\
\hline \multicolumn{7}{|l|}{ Yes } \\
\hline \multicolumn{7}{|l|}{$\mathrm{No}^{\mathrm{a}}$} \\
\hline Method of length determination & 0.332 & 0.402 & 0.684 & 1 & $1.394(0.634-3.063)$ & 0.408 \\
\hline \multirow{2}{*}{\multicolumn{7}{|c|}{$(\mathrm{NEX} \times 0.38696)+30.37^{\mathrm{a}}$}} \\
\hline & & & & & & \\
\hline Awareness level ${ }^{\mathrm{a}}$ & & & 0.634 & 2 & & 0.728 \\
\hline Awareness level(1) & 0.191 & 0.442 & 0.187 & 1 & $1.211(0.509-2.883)$ & 0.665 \\
\hline Awareness level(2) & 0.542 & 0.695 & 0.609 & 1 & $1.720(0.441-6.711)$ & 0.435 \\
\hline Constant & -4.400 & 6.438 & 0.467 & 1 & 0.012 & 0.494 \\
\hline
\end{tabular}

CI, confidence interval; df, degrees of freedom; NEX, nose-earlobe-xiphoid distance; OR, odds ratio.

${ }^{\mathrm{a}}$ Reference category.

lead to regurgitation and an increased risk of pulmonary aspiration. ${ }^{14}$

The NEX method is the most commonly used technique for measuring the nasogastric tube length but has been questioned in the literature. ${ }^{8-10}$ After a postmortem study including 99 cases, Hanson concluded that the NEX method resulted in $72 \%$ well-placed nasogastric tubes, with the ideal tip position between 1 and $10 \mathrm{~cm}$ under the LES. ${ }^{2}$ Using the 2 Hanson formulas adjusting the NEX, the number of properly placed tubes increased to around $92 \%$; however, approximately $3 \%$ still were located in the distal esophagus, which was about the same for the NEX $(2 \%)$. Note that Hanson's study did not mention any lateral openings in the nasogastric tubes, now common in most tubes. Therefore, the tube must be inserted sufficiently deep into the stomach to ensure that not only the tip but also all lateral openings are located inside the stomach to prevent tube feeding entering the esophagus. In our study, the presence of lateral openings may explain why the percentage of insufficiently deep placed nasogastric tubes exceeded Hanson's. Hanson also stated that the NEX might lead to an overestimation of the required length, resulting in a placement $>10 \mathrm{~cm}$ under the LES in $25 \%$ of cases compared with $<6 \%$ using Hanson's formulas. Our study reached the same conclusion, with $17.2 \%$ of the tubes located $>10 \mathrm{~cm}$ under the LES in the control group and $4.8 \%$ in the intervention group.

In a study with a cross-sectional design, Ellett et al tested the reliability of 1 of the formulas proposed by Hanson $(([\mathrm{NEX}-50 \mathrm{~cm}] / 2)+50 \mathrm{~cm})$ compared with the NEX. ${ }^{3}$ A correct tip position was defined as between 3 and $10 \mathrm{~cm}$ under the LES. In the NEX group, 9 out of 21 malpositioned tubes were placed insufficiently deep vs 11 of 19 tubes in the Hanson group. Additionally,
24 potentially predicting variables were included in the data collection and analysis in order to develop a new method for external length determination. This resulted in a 3-variable model of calculation based on gender, weight, and the distance between nose and umbilicus in supine position with the head flat (GWNUF), in which gender was " 1 " if the participant was male and " 0 " if the participant was female: $29.38+(4.53 \times$ gender $)+(0.34 \times \mathrm{NUF})-$ $(0.06 \times$ weight $)$. The GWNUF resulted in $85.3 \%$ of correct positions (vs $72 \%$ for the NEX and $84.2 \%$ for Hanson) but here also 5 of 11 malpositioned tubes were in the esophageal danger zone. In addition to increasing the risk of feeding in the esophagus due to a malpositioned tip of the nasogastric tube, the feasibility of the GWNUF in clinical practice can also be questioned for other reasons: the difficulty of obtaining the correct weight of bedridden patients; discomfort for the patients to maintain the required position; and higher likelihood to make clinical errors when using different parameters to estimate the insertion length in clinical practice. The combination of these elements, together with the large number of included ICU patients in the study, ensured that this method was not selected as the preferred intervention in the study. It may not possible to obtain an accurate weight in critically ill patients who usually are also ventilated. In addition, the positioning of ICU patients as described above is not always possible due to their physical condition.

The study of Boeykens et al. (2014) mainly focused on $\mathrm{pH}$ measurement as an alternative method for $\mathrm{X}$-ray to confirm the correct position of the nasogastric tube. ${ }^{12}$ In Boeykens' study, the probability of a correctly placed nasogastric tube was $98.9 \%$ when taking $\mathrm{pH}$ measurement as criterion. Metheny et al stated that obtaining 
gastric aspirate and $\mathrm{pH}$ measurement is not useful to detect placement of the nasogastric tube in the esophagus because of possible gastric reflux. ${ }^{15,16}$ Therefore, caution should be exercised in interpreting a $\mathrm{pH}$ measurement on gastric aspirate. Our study showed that a correctly positioned tip inside the stomach increased the possibility to obtain gastric aspirate by almost 4 times. In our study, $>20 \%$ of all nasogastric tubes were placed too shallow whether the NEX or Hanson's formula was used to determine the insertion length. Therefore, it is recommended to combine either method for external length determination with X-ray to verify correct placement of the tip.

Taylor et al suggested the use of NEX $+10 \mathrm{~cm}$ as an alternative to the NEX method, which would lead to a reduction from $16 \%$ to $7 \%$ of misplaced nasogastric tubes, with the tip located into the esophagus. This small study $(\mathrm{N}=36)$ using electromagnetic guidance and not $\mathrm{X}$-ray was solely conducted in an intensive care setting in a population of mainly neurosurgery and trauma patients with gastric emptying problems whose stomachs possibly dilate more than in other patients. ${ }^{5}$ This method made it possible to guide the tip of the tube in the stomach immediately. In earlier studies ${ }^{2,3}$ as well as in this current study, an overestimation of the NEX was observed; thus, the NEX $+10 \mathrm{~cm}$ potentially could result in tubes migrating postpyloric, curling up/kinking inside the stomach—or even worse, migrating up and re-entering the esophageal danger zone.

There are some limitations in this prospective randomized trial. Firstly, there was no registration of the number of excluded patients with previously documented hiatus hernia during the period of data collection. After including all patients in the study, there was also a $14.9 \%$ patient dropout due to the poor quality of the chest X-ray images (tube or tip not visible). Secondly, there was no separate registration of data obtained by the 2 clinical nutrition nurse specialists measuring the NEX distances, and thus no conclusions can be drawn about interrater variability. Finally, the $\kappa$ value $(\kappa=0.44)$ of the interobserver agreement between the radiologists can also be questioned. The clinical significance of this value depends on its context. In a review ${ }^{17}$ of medical imaging literature regarding imaging for expressing observer agreement with regard to categorical data, the conclusion was made that a $\kappa$ value $\geq 0.4$ could be considered an acceptable level of observer variability. For this reason, the classification system used by Coblentz at al., in which agreement is quantified as poor, fair, moderate, good, or excellent, was followed. ${ }^{13}$

Despite the fact that Hanson's formula in our study led to $72.6 \%$ correctly placed nasogastric tubes, with the tip located somewhere between 3 and $10 \mathrm{~cm}$ under the LES, there were still outliers (both over- and underestimation of the insertion length) that could not be associated with any of the variables of the data collection. Despite all, this study again emphasizes the importance of strict and clear guidance on preventing potential incidents that may lead to harm or death. Therefore, further research should focus on determining other possible influencing factors (e.g.k anatomical characteristics) for a more correct gastric tip positioning in order to develop an easy, safe, and clinically feasible method for estimating the insertion length of nasogastric tubes.

\section{Conclusions}

Both the NEX-method and Hanson's formula are not reliable to determine the appropriate length of the nasogastric tube in order to obtain a correct tip position between 3 and $10 \mathrm{~cm}$ under the LES. At the same time, differences in the likelihood of obtaining gastric aspirate could not be demonstrated between the 2 methods. In $>20 \%$ of patients, the tip of the nasogastric tube was located in the esophageal danger zone. To reduce the risk of misplacement, and consequently also reduce the inconvenience caused to patients due to repositioning of the tube, a more adequate method of determining the appropriate length of the nasogastric tube should be developed. Meanwhile, it is strongly recommended to confirm placement of the tube tip through X-ray and reposition the tube when necessary. This will minimize the risk of feeding remaining in the esophagus and of possible reflux.

\section{Statement of Authorship}

T. Torsy, R. Saman, K. Boeykens and I. Duysburgh equally contributed to the conception and design of the research; D. Beeckman and N. Van Damme contributed to the design of the research; T. Torsy, R. Saman, K. Boeykens and N. Van Damme contributed to the acquisition and analysis of the data; T. Torsy, D. Beeckman and N. Van Damme contributed to the interpretation of the data; and T. Torsy and R. Saman drafted the manuscript. All authors critically revised the manuscript, agree to be fully accountable for ensuring the integrity and accuracy of the work, and read and approved the final manuscript.

\section{Aknowledgements}

The authors would like to thank Leo Verguts, Karline Schutyser, Dirk De Backer, Kurt Van Belle and Patrick Palmans for their valuable contribution to this study and Leen Trommelmans for assisting with the final draft. They also would like to thank Odisee University College and the general hospital AZ Nikolaas for facilitating this study.

\section{References}

1. Ferrua MJ, Singh RP. Modeling the fluid dynamics in a human stomach to gain insight of food digestion. J Food Sci 2010;75: 151-162.

2. Hanson RL. Predictive criteria for length of nasogastric tube insertion for tube feeding. JPEN J Parenter Enteral Nutr 1979;3:160-163. 
3. Ellett MLC, Beckstrand J, Flueckiger J, Perkins SM, Johnson CS. Predicting the insertion distance for placing gastric tubes. Clin Nurs Res 2005;14:11-27.

4. Chen Y, Wang L, Chang Y et al. Potential risk of malposition of nasogastric tube using nose-ear-xiphoid measurement. PLoS One 2014;9:e88046.

5. Taylor SJ, Allan K, McWilliam H, Toher D. The 'NEX' guideline is incorrect. Br J Nurs 2014;23:641-644.

6. National Patient Safety Agency (NPSA). Patient Safety Alert: Nasogastric tube misplacement: continuing risk of death and severe harm. Updated July 22, 2016. Available at: https://improvement.nhs.uk/ news-alerts/nasogastric-tube-misplacement-continuing-risk-of-deathsevere-harm. Accessed March 2, 2018.

7. Fitzpatrick JJ, Wallace M. Encyclopaedia of Nursing Research 3rd ed. New York, NY: Springer Publishing; 2011.

8. Santos SC, Woith W, Freitas MI, Zeferino EB. Methods to determine the internal length of nasogastric feeding tubes: an integrative review. Int J Nurs Stud 2016;61:95-103.

9. Beckstrand J, Ellett MLC, McDaniel A. Predicting the internal distance to the stomach for positioning nasogastric and orogastric feeding tubes in children. $J$ Adv Nurs 2007;59:274-289.
10. Ellett MLC, Cohen MD, Perkins SM, Smith CE, Lane KA, Austin JK. Predicting the insertion length for gastric tube placement in neonates. J Obstet Gynecol Neonatal Nurs 2011;40:412-421.

11. Sealed Envelope Ltd. Create a blocked randomisation list. Updated January 1, 2015. Available at: https://www.sealedenvelope.com/simplerandomiser/v1/lists. Accessed October 30, 2015.

12. Boeykens K, Steeman E, Duysburgh I. Reliability of $\mathrm{pH}$ measurement and the auscultatory method to confirm the position of a nasogastric tube. Int J Nurs Stud 2014;51:1427-1433.

13. Coblentz CL, Babcook CJ, Alton D, Riley BJ, Norman G. Observer variation in detecting the radiologic features associated with bronchiolitis. Invest Radiol 1991;26:115-118.

14. Metheny NA, Titler MG. Assessing placement of feeding tubes. Am J Nurs 2001;101:36-45.

15. Metheny NA, Reed L, Wiersema L, McSeeney M, Wehrle MA, Clark J. Effectiveness of $\mathrm{pH}$ measurements in predicting feeding tube placement: an update. Nurs Res 1993;42:324-331.

16. Metheny NA, Clouse RA, Clarck JM, Reed L, Wehrle MA, Wiersema L. $\mathrm{pH}$ testing of feeding-tube aspirates to determine placement. Nutr Clin Pract 1994;9:185-190.

17. Kundel HL, Polansky M. Measurement of observer agreement. Radiology 2003;228:303-308. 